\title{
Historia y teoría de la recepción
}

Peter Burke*

Quidquid recipitur, ad modum recipientis recipitur.

Thomas Aquinas

En lo que sigue argumentaré (1) que el concepto de recepción (en alemán Rezeption, en francés réception, en italiano recezione, etc.), es más antiguo de lo que generalmente se piensa; (2) que, aún así, el reciente 'giro' en esta dirección resulta muy significativo; (3) que el concepto de 'traducción cultural' resulta útil para los estudios de recepción; (4) que una serie de problemas asedian a los académicos que intentan rastrear la historia de la recepción de textos u otros artefactos culturales; y que, a pesar de estos problemas, este enfoque sigue siendo esclarecedor para la historia intelectual. Sobre la base de que cuanto más distantes están las dos culturas más visible se vuelve el proceso de recepción, privilegiaré ejemplos provenientes de las relaciones culturales entre Europa y el este asiático.

Como sucede con tantas otras ideas aparentemente nuevas, la idea de recepción tiene una historia más larga de lo que podríamos pensar. Especialmente en Alemania, estaba presente como término hace unos cien años, en los círculos eruditos de estudio sobre derecho romano y humanismo renacentista. ${ }^{1}$ El término

* Publicado originalmente como "Chapter One: The History and Theory of Reception", en Howell A. Lloyd (ed.), The Reception of Bodin, Leiden, Brill, 2013, 21-37. Traducción por Lucas Domínguez Rubio con derechos de publicación y traducción adquiridos por el CeDInCl. Agradecemos especialmente la amabilidad de Peter Burke y Howell A. Lloyd por permitirnos traducir y publicar este artículo.

1 Carl Adolf Schmidt, Die Reception des Römischen Rechts in Deutschland (1868), Leipzig, Zentralantiquariat der DDR, 1969; Paul Laband, Rede über die Bedeutung der Rezeption des römischen Rechts für das deutsche Staatsrecht, Strasbourg, University of Strasbourg, 1880; Max Herrmann, Die Reception des Humanismus in Nürnberg, Berlin, Wiedmann, 1898; Stefan Schuler, Vitruv im Mittelalter: Die Rezeption von "De Architectura" von der Antike bis in die frühe Neuzeit, Cologne, Böhlau, 1999. también fue utilizado por los académicos dedicados a la literatura de habla inglesa y, un poco después, por algunos historiadores de la religión. ${ }^{2}$

La idea de recepción también atrajo el interés de estudiantes de literatura, arte e ideas que no hicieron uso de esta palabra. En particular, los clasicistas hablaron de 'tradición'. ${ }^{3}$ En Alemania, un término alternativo fue 'otra vida' (Nachleben o Fortleben). ${ }^{4}$ En Italia, el término preferido fue y es fortuna. ${ }^{5}$ Algunos autores ingleses prefirieron, y de hecho todavía prefieren, 'influencia', especialmente en los estudios literarios, pero también en la historia intelectual y en la historia del arte. ${ }^{6}$ Otros eligieron, y siguen eligiendo, el término 'legado'?

La mayoría sino todos los estudios mencionados hasta ahora han

2 William Frederic Hauhart, The Reception of Goethe's Faust in England in the First Half of the 19th Century, New York, Columbia University Press, 1909; Lawrence M. Price, The Reception of English Literature in Germany, Berkeley, University of California Press, 1932; Thomas A. Brady, The Reception of Egyptian Cults by the Greeks, Columbia, University of Missouri, 1935; Charles D. Cremeans, The Reception of Calvinistic Thought in England, Urbana, University of Illinois Press, 1949.

3 Anthony Grafton, Glenn W. Most y Salvatore Settis (eds.), The Classical Tradition, Cambridge, Harvard University Press, 2010.

$4 \quad$ Otto Immisch, Das Nachleben der Antike, Leipzig, Dieterich, 1919; Jacob Walter, Wiliam Blakes Nachleben in der englischen Literatur des neunzehnten und zwanzigsten Jahrhunderts, Schaffhausen, Bachmann, 1927.

5 Por ejemplo: Vincenzo Luciani, Francesco Guicciardini e la fortuna deIl'opera sua, Florence, Olschki, 1949; Giuliano Procacci, Studi sulla fortuna del Machiavelli, Rome, Istituto Storico Italiano per l'età moderna e contemporanea, 1965.

6 Alfred E. Taylor, Platonism and its Influence, London, Harrap, 1925; Thomas F. Scanlon, The Influence of Thucydides on Sallust, Heidelberg, Winter, 1980; Henry H. Reed, Palladio's Architecture and its Influence, New York, Dover, 1980; Mordechai Feingold, Joseph S. Freeman y Wolfgang Rother (eds.), The Influence of Petrus Ramus, Basel, Schwabe, 2001.

7 Richard W. Livingstone (ed.), The Legacy of Greece, Oxford, Clarendon Press, 1921; Cyril Bailey (ed.), The Legacy of Rome, Oxford, Clarendon Press, 1923; Joseph V. Femia (ed.), The Machiavellian Legacy, Basingstoke, Macmillan, 1998. 
analizado la recepción esencialmente desde el punto de vista del autor o productor, tratando a los receptores como relativamente pasivos, es decir, como seguidores: maquiavélicos, erasmianos, luteranos y demás. Términos como 'transmisión' o 'transferencia' (como en el caso de 'transferencia de tecnología') también enfatizan el papel del productor. ${ }^{8}$ Los estudios de recepción, transferencia, tradición y legado generalmente dependen de la asunción de fidelidad o continuidad, dando por sentado que lo que se ha recibido o heredado era lo mismo que lo que fue dado o entregado. En este sentido, los académicos siguen a quienes estudiaban, desde la antigüedad clásica hasta los tempranos tiempos modernos, cuando los términos preferidos fueron traditio y traslatio (en el sentido de 'transferencia').

Por supuesto las tradiciones pueden ser criticadas como corruptas, al igual que los reformadores criticaron las tradiciones de la Iglesia Católica, pero la crítica implicaba que la purificación o el retorno ad fontes era posible. Una observación similar podría hacerse sobre la crítica de algunas traducciones consideradas infieles, como en el famoso debate en la Francia del siglo XVII sobre les belles infidèles. ${ }^{9}$ Aunque el famoso epigrama atribuido a Karl Marx, "No soy marxista", ha circulado durante mucho tiempo, las implicaciones sobre la distancia entre los fundadores y los seguidores rara vez se ha hecho explícita para la historia intelectual. ${ }^{10}$

Algunos estudiosos han encontrado fallas en estos enfoques, específicamente en el concepto de 'influencia'. Tan temprano como en 1945, R. G. Collingwood criticó lo que llamó "el tipo frívolo y superficial de historia que habla de 'influencias' y 'préstamos' y nunca se pregunta sobre qué había en A que lo dejaba abierto a la influencia de $B$, o qué había en $A$ que lo hiciera capaz de pedir prestado a B". Quentin Skinner hizo una crítica similar en 1969 y Michael Baxandall en 1985: "'Influencia' es una maldición en la crítica de arte, principalmente debido a su prejuicio gramatical equivocado sobre quién es el agente y quién el paciente... Si uno dice que $\mathrm{x}$ influyó en $\mathrm{y}$, parece que está diciendo que $\mathrm{x}$ le hizo algo a y en lugar de que y le hizo algo a x... Si pensamos en y en lugar de $x$ como el agente, el vocabulario resulta más rico, atractivo y diversificado: utilizar, recurrir, aprovecharse, apropiarse, apoyarse, adaptar, malinterpretar, referirse"."

8 Michel Espagne, Les Transferts culturels franco-allemands, Paris, Presses Universitaires de France, 1999.

9 Jean-Pierre Massaut, Critique et tradition à la veille de la Réforme en France, Paris, Vrin, 1974; Roger Zuber, Les "belles infidèles" et la formation du goût classique, Paris, Colin, 1968.

10 Para tener en cuenta una importante excepción, ver Benjamin Schwartz, "Some Polarities in Confucian Thought," en David Nivison and Arthur Wright (eds.), Confucianism in Action, Chicago, University of Chicago Press, 1959.

11 Robin G. Collingwood, The Idea of Nature, Oxford, Oxford University Press, 1945, p. 128. Ver: Quentin Skinner, "Meaning and Understanding in the History of Ideas", en History and Theory, $n^{\circ} 8,1969$, pp. 3-53; Michael Baxandall, Patterns of Intention, New Haven, Yale University Press, 1985, pp. 58-9. Skinner y Baxandall (como Jauss, discutido a continuación) citan a Collingwood con aprobación.
Como si se tratara de una respuesta a estas críticas, a finales del siglo xx se registró una nueva ola por — no decir una inundación- de estudios de recepción. Lo importante no era tanto el aumento del número, la creación de una tendencia o, como dirían los críticos, una "moda" académica, sino el cambio en la forma en que se consideraba a los seguidores o receptores. En lugar de ser considerados como receptores pasivos de "influencias" o en el mejor de los casos como carentes de ideas propias, se les concedió el carácter de agentes y el énfasis se centró en los usos o respuestas, desde el punto de vista del receptor. Estos cambios en el lenguaje constituyen un indicador sensible de la nueva tendencia presente en estos estudios. En particular, los académicos que estudian el renacimiento se encontraron utilizando más y más palabras que comienzan con 're': no sólo 'recepción', sino también 'relectura', 'reescritura', 'reempleo', 'reencuadre', 'reinterpretación' y 'recontextualización'.12

En los estudios literarios, este giro está asociado al auge de la "teoría de la recepción" y en particular con dos teóricos alemanes, Hans-Robert Jauss y Wolfgang Iser.13 Jauss subrayó lo que él Llamó la "estética de la recepción", mientras que Iser enfatizó la "respuesta del lector", y consideró el significado como el producto de la interacción entre el lector y el texto, pero a ambos se los ha vinculado conjuntamente como responsables de la Escuela de Constanza, la nueva universidad alemana en donde enseñaron. La obra de Iser en particular se ha hecho muy conocida en el mundo literario de habla inglesa, gracias en parte a su nombramiento como profesor en la Universidad de California de Irvine a finales de los años setenta.

Quizás la característica más distintiva de este enfoque es la preocupación por el 'horizonte de expectativas' (Erwartungshorizont), el argumento consiste en que las diferentes expectativas que aportan los distintos lectores dan forma a las maneras en que se entiende un texto determinado. Para dar un ejemplo proveniente de la historia intelectual, un estudio sobre la ilustración alemana sostiene que, en las décadas de 1770 y 1780, James Steuart fue tomado más seriamente como escritor en economía que Adam Smith, y destaca la atracción que tuvo su trabajo An Inquiry into the Principles of Political Economy para lectores acostumbrados a las ideas de escritores alemanes como J. H. G.

12 Maryanne C. Horowitz, Anne J. Cruz y Wendy A. Furman (eds.), Renaissance Rereadings, Urbana, University of Illinois Press, 1988; Centre Interuniversitaire de recherche sur la Renaissance italienne, Réécritures: commentaires, parodies, variations dans la literature italienne de la Renaissance (3 vols.), Paris, Université de la Sorbonne Nouvelle, 1983 p. 7; Claire Farago (ed.), Reframing the Renaissance, New Haven, Yale University Press, 1995.

13 Hans-Robert Jauss, Literaturgeschichte als Provokation, Frankfurt, Suhrkampf, 1970; Wolfgang Iser, Der Akt des Lesens: Theorie ästhetischer Wirkung, Munich, Fink, 1976; Robert C. Holub, Reception Theory: a Critical Introduction, London, Methuen, 1984, sigue siendo una guía útil. 
Justi sobre el mismo tema. ${ }^{14}$ La metáfora de 'horizonte' es una metáfora tradicional en la filosofía alemana, transmitida de Edmund Husserl a su estudiante Martin Heidegger, luego al estudiante de Heidegger, Hans-Georg Gadamer, y luego al estudiante de Gadamer, Iser —aunque no siempre fue empleada de la misma manera por estos cuatro pensadores. Otro concepto común a Gadamer, Jauss e Iser es el de Wirkung, que se refiere al efecto de un mensaje en los lectores (u oyentes). Sin embargo, la distinción entre Rezeption yWirkung aún sigue sin estar clara. ${ }^{15}$

En el sentido estricto del término, 'teoría de la recepción' se refiere a este enfoque teórico de origen alemán. Sin embargo, en Francia hubo un movimiento paralelo más amplio que no estuvo confinado a los estudios literarios. Las mayores figuras de este movimiento fueron el filósofo Paul Ricoeur y el polifacético erudito Michel de Certeau. Ambos destacaron el papel de los receptores como agentes. Con este propósito, Ricoeur propuso el concepto de 'apropiación', un término que tal vez debería preferirse al de 'recepción', precisamente porque se lo asocia con la actividad más que con la pasividad. En cuanto a De Certau, su concepto favorito fue el de 'reempleo' (ré-emploi). Al reaccionar contra el enfoque sociológico usual que consideraba a la gente común como consumidora pasiva de los bienes producidos en masa, De Certau argumentó que, después de todo, los individuos ejercen su libertad cuando seleccionan qué comprar y cómo usarlo, de modo que, al combinar su compra con otros objetos, adaptan y personalizan sus adquisiciones. Haciendo referencia a la idea de Claude Claude Lévi-Strauss de bricolage intelectual, pero desarrollándola más, Certau enfatizó que el consumo debe ser considerado como una forma de producción. ${ }^{16}$

En los estudios literarios, Julia Kristeva lanzó la idea de intertextualidad para enfatizar los modos en que un texto refiere a otros (por imitación, por refutación, por parodia, etc.), en otras palabras, para enfatizar el bricolage literario, mientras que Gérard Genette desarrolló esta idea y distinguió entre transtextualidad, metatextualidad, paratextualidad, etc.. ${ }^{17}$

Todavía los estudios sobre recepción siguen despertando más intereses en los departamentos de literatura que en los demás lugares del campus, con la posible excepción de los departamentos de comunicación, especialmente después del reconocido estudio

14 Keith Tribe, Governing Economy: the Reformation of German Economic Discourse, 1750-1840, Cambridge, Cambridge University Press, 1988, p. 140.

15 Holub, Reception Theory, op. cit., p. xi.

16 Paul Ricoeur, "Appropriation", en Ricoeur, Hermeneutics and the Human Sciences: Essays on Language, Action and Interpretation, Cambridge, Cambridge University Press, 1981, pp. 182-93; Michel de Certeau, L'invention du quotidien, Paris, Union Générale d'Éditions, 1980.

17 Julia Kristeva, "Bakhtine, le mot, le dialogue et le roman", en Critique ${ }^{\circ}$ 239, 1967, pp. 438-65. Ver: Michael Worton y Judith Still (eds.), Intertextuality: theories and practices, Manchester, Manchester University Press, 1990, especialmente las páginas 1-44; Graham Allen, Intertextuality, London, Routledge, 2000, especialmente: pp. 30-60 y pp. 97-115. sobre la recepción de la telenovela Dallas en diferentes partes del mundo, que señaló que las mismas imágenes vistas por diferentes grupos no podrían haber sido entendidas de manera más diferente. ${ }^{18}$

También en otros campos de estudios existe un interés creciente. Por ejemplo, los historiadores del arte estudian la recepción de pintores o tipos de arte particulares (como, por ejemplo, el arte japonés en Occidente), y algunos historiadores de la arquitectura están interesados en el reempleo en un sentido más literal que Certau, mediante la utilización de fragmentos de viejas construcciones en otras nuevas. ${ }^{19}$ Los historiadores dedicados a la historia intelectual también se han movido en esta dirección. Heidegger y Jauss estuvieron entre los inspiradores de la 'historia conceptual' (Begriffsgeschichte) practicada por Reinhart Koselleck y desarrollada en los enormes volúmenes de la obra Geschichtliche Grundbegriffe. En particular, Koselleck hizo un uso frecuente de la idea de horizonte de expectativas. ${ }^{20}$ En términos generales, se volvió visible un cambio alrededor de la recepción en la historia de la ciencia, la historia del pensamiento político y la historia de la religión. En el caso de la historia de la ciencia, el darwinismo y el copernicanismo han atraído una atención particular. ${ }^{21}$ En el campo del pensamiento político, algunos estudios sobre el pensamiento de John Locke ejemplifican esta nueva tendencia. ${ }^{22}$

En el caso de la religión, se podrían comparar y contrastar dos estudios sobre Erasmo y sus seguidores que fueron publicados con medio siglo de diferencia. En su estudio sobre Erasmo y sus escritos en la España del siglo XVI, Marcel Bataillon escribió en términos de érasmisme, mouvement érasmien, evangélisme érasmien y así sucesivamente, haciéndose la pregunta fundamental para los estudios de recepción, ¿por qué aquí? “¿Cómo fue que esta forma erasmiana de cristianismo floreció más espectacularmente en España que en cualquier otra lado?". Cincuenta años después, Silvana Seidel Menchi produjo una importante monografía sobre los lectores de Erasmo en Italia que a la vez siguió y difirió con el trabajo de Bataillon. A diferencia de Bataillon, Seidel Menchi utilizó el término ricezione y, en sintonía con los recientes estu-

18 Tamar Liebes y Elihu Katz, The Export of Meaning: Cross-Cultural Readings of Dallas, New York, Oxford University Press, 1990.

19 Elisa Evett (ed.), The Critical Reception of Japanese Art in Late Nineteenth-Century Europe, Epping, Bowker, 1982; Lucilla de Lachenal, Spolia: uso e reimpiego dell'antico dal III al XIV secolo, Milan, Longanesi, 1995.

20 Otto Brunner, Werner Conze y Reinhart Koselleck (eds.), Geschichtliche Grundbegriffe (8 vols.), Stuttgart, Klett, 1972, p. 97. Las nociones de recepción y horizonte son discutidas en Reinhart Koselleck, Futures Past: on the Semantics of Historical Time (trad. Keith Tribe), Cambridge, MIT Press, 1985, pp. 7, 46, 56, 64, 106, 186, 196-7, 200, 267-88.

21 Thomas F. Glick (ed.), The Comparative Reception of Darwinism, Austin, University of Texas Press, 1974; Rienk Vermij, The Calvinist Copernicans: the Reception of the New Astronomy in the Dutch Republic, 1575-1750, Amsterdam, Koninklijke Nederlandse Akademie van Wetenschappen, 2002.

22 Mark Goldie (ed.), The Reception of Locke's Politics, London, Pickering and Chatto, 1999. 
dios de recepción, criticó el término erasmismo como "una categoría desgastada por el exceso de uso", para en contraposición remarcar el uso que los italianos, que tenían su propia agenda, realizaron de Erasmo, o bien con el fin de disfrazar las creencias protestantes o bien con el de legitimar un ataque político contra el papado. ${ }^{23}$

También hay movimientos paralelos o conectados en otras disciplinas. Por ejemplo, los clasicistas están examinando la tradición griega y romana a través de los lentes de la recepción. ${ }^{24}$ Los historiadores y los críticos literarios ahora prestan más atención a los lectores y sus respuestas en comparación a lo que solían hacer. ${ }^{25}$ Un estudio reciente sobre la suerte de Cervantes en la Inglaterra del siglo xvii comienza con la pregunta, "¿qué percibieron los primeros lectores anglo-parlantes de Don Quijote?" y continúa haciendo notar que los escritores ingleses "pusieron a Don Quijote a trabajar para sus propios fines". ${ }^{26}$

Certau fue una de las inspiraciones de la investigación de Roger Chartier para su historia de la lectura. También algunos historiadores han estudiado los comentarios suscitados por los textos de Castiglioni, Montaigne, Copérnico y otros escritores para analizar su recepción. ${ }^{27}$ De manera similar, los historiadores del arte han puesto el foco en los espectadores y los musicólogos en escuchar a los oyentes. ${ }^{28}$ Los historiadores de la economía también prestan más atención a los consumidores que antes, mientras que muchos historiadores sociales, e incluso de la política, han girado hacia la historia 'desde abajo', para enfatizar el accionar de la gente común.

23 "Comment ce christianisme érasmien a-t-il fleuri en Espagne plus brillament qu'ailleurs?", en Marcel Bataillon, Erasme en Espagne (1937) Geneva, Droz, 1991, p. 846; Silvana Seidel Menchi, Erasmo in Italia: 1520-1580, Turin, Bollati Boringhieri, 1987.

24 Charles Martindale, Redeeming the Text: Latin poetry and the Herme neutics of Reception, Cambridge, Cambridge University Press, 2003; Martindale y Richard F. Thomas (eds.), Classics and the Uses of Reception, Oxford, Blackwell, 2006; Philip Ford, De Troie à Ithaque; réception des épopées homériques à la Renaissance, Geneva, Droz, 2007.

Iser, Akt des Lesens; Susan Suleiman e Inge Crosman (eds.), The Reader in the Text, Princeton, Princeton University Press, 1980; Robert Darnton, "History of Reading," en Peter Burke (ed.), New Perspectives on Historical Writing, Cambridge, Polity Press, 2001, pp. 157-86; Guglielmo Cavallo y Roger Chartier (eds.), A History of Reading in the West (trad. Lydia G. Cochrane), Amherst, University of Massachusetts Press, 1999.

Dale B. J. Randall y Jackson C. Boswell, Cervantes in Seventeenth-Century England: the Tapestry Turned, Oxford, Oxford University Press, 2009, pp. xv, xxxvii.

27 Roger Chartier, Cultural History between Practices and Representations (trad. Lydia G. Cochrane), Cambridge, Polity Press, 1988, pp. 40 1; Peter Burke, The Fortunes of the Courtier, Cambridge, Polity Press 1995; Warren Boutcher, "Marginal Commentaries: the Cultural Transmission of Montaigne's Essais in Shakespeare's England", en Pierre Kapitaniak y Jean-Marie Maguin (eds.), Shakespeare et Montaigne, Paris, Société Française Shakespeare, 2003, pp. 13-27; Owen Gingerich, The Book Nobody Read: Chasing the Revolutions of Nicolaus Copernicus New York, Walker, 2004

28 Wolfgang Kemp (ed.), Der Betrachter ist im Bild, Cologne, DuMont 1985; David Freedberg, The Power of Images, Chicago, University of Chicago Press, 1989; James H. Johnson, Listening in Paris: a cultural history, Berkeley, University of California Press, 1995.
Como sucede tan frecuentemente en la historia del pensamiento histórico, los cambios en el presente, desde el populismo hasta la preocupación por el medioambiente, han alentado a los académicos a hacerse diferentes preguntas sobre el pasado.

Por ejemplo, en el caso de los clásicos, donde especialmente en la última década los estudios de recepción han ganado mucho terreno, la necesidad de responder al declive del estudio del griego y el latín resulta suficientemente obvia. El creciente interés en el nuevo empleo metafórico de la Odisea o la Eneida podría interpretarse de manera injusta como una reacción a la necesidad literal del re-empleo de los ex-clasicistas.

A diferencia de los estudios de recepción de alrededor del año 1900, el nuevo movimiento es o al menos fue, atrás en los años setenta subversivo, chocante e incluso escandaloso, porque cambiaba el énfasis desde la pasividad y la fidelidad de la recepción hacia una recepción activa o creativa. Los académicos dedicados a los estudios de recepción colaboraron en desenmascarar la ilusión de la comunicación perfecta, en tanto socavaron la importancia de la intención de los autores, artistas y filósofos, y cuestionaron la existencia de un significado fijo. Algunos de los académicos involucrados en este movimiento no tuvieron tales ambiciones, pero, de modo adecuado en este contexto, sus estudios fueron recibidos de este manera.

A pesar de que para algunos críticos el enfoque de la recepción parecía chocante, el énfasis en los lectores y espectadores como apropiadores no era completamente nuevo. El concepto de 'préstamo' cultural es muy antiguo y enfatiza el papel de los receptores, aunque a menudo se suponía que lo que se tomaba prestado era idéntico a lo que se prestaba. Por esta razón, cuando escribió sobre el Renacimiento en los años veinte, el iconoclasta Lucien Febvre rechazó el concepto de préstamo, sobre la base de que los artistas y escritores de aquel tiempo "han combinado, adaptado, transpuesto" produciendo "algo que era construido y original al mismo tiempo".29

Algunos escritores brasileños hicieron un comentario similar más o menos al mismo tiempo, incluso de forma más vívida. En su Manifesto antropófago (1928), Oswald de Andrade jugó con el estereotipo europeo sobre los brasileños como caníbales, para preguntarse si los escritores como él mismo deberían o no seguir los modelos europeos. Oswald atacó lo que él llamó "importadores de conciencia enlatada" y sugirió que los brasileños eran capaces de digerir ideas extranjeras haciéndolas suyas. ${ }^{30}$

De hecho, la suposición de fidelidad había sido desafiada mucho

29 "ont combiné, adapté, transposé": "quelquechose de composite et d'original à la fois", Lucien Febvre, "La Première Renaissance française," Pour une histoire à part entière, Paris, Sevpen, 1962, pp. 529-603.

30 Carlos A. Jauregui, Canibalia. Canibalismo, calibanismo, antropofagia cultural y consumo en América Latina, Madrid, Iberoamericana, 2008. 
antes por Tomás de Aquino. En su famosa Summa Theologiae, Aquino formuló el principio Quidquid recipitur, ad modum recipientis recipitur: "todo lo que se recibe es recibido de acuerdo al modo del receptor" ${ }^{31}$ Como bien sabía Tomás de Aquino, algunos de los Padres de la Iglesia (Basilio, Orígenes, Jerónimo y Agustín) habían respondido en forma similar a un problema difícil: ¿qué se debía hacer con las tradiciones paganas dentro de un mundo cristiano? Su solución fue enfatizar lo que ahora llamamos apropiación selectiva, o en su vocabulario mucho más vívido, "botín" (spolia). Agustín, citando Exodus iii.22, "spoliabitis Egyptum", comparó el uso cristiano de los clásicos con el pueblo de Israel que saqueaba el tesoro de Egipto cuando se marchaba. Por su parte, Orígenes y Jeremías, citando Deuteronomium xxi.11-13, utilizaron la chocante metáfora sexista sobre la bella cautiva: los lectores cristianos podían hacer que los clásicos paganos sirvieran a sus propios propósitos, así como los israelitas habían utilizado a las mujeres egipcias capturadas y esclavizadas por ellos, cortándoles el pelo y las uñas. ${ }^{32}$ Basilio de Cesarea usó la metáfora de las abejas que "ni se acercan a todas las flores por igual, ni tratan de llevarse las que eligen enteras, sino que sólo toman lo que es adecuado para su trabajo y dejan el resto intacto". 33

Los padres de la iglesia constituyen un puente hacia la teoría de la recepción porque dos de los principales teóricos franceses, Paul Ricoeur y Michel de Certau, fueron buenos lectores de la patrística. La discusión de Ricoeur sobre la apropiación, así como la presentación de la noción de reempleo por De Certeau, fueron en sí mismas una traducción o adaptación creativa de las ideas de Agustín y Jeremías. En su estudio de recepción más conocido, De Certau se refería a los lectores como lo había hecho Agustín, "saqueadores de los bienes de los egipcios para disfrutarlos ellos mismos". ${ }^{34}$

\section{3}

Existen otras tradiciones de estudios de recepción más allá de la alemana y la francesa. En los estudios literarios de los Estados Unidos, el trabajo de Harold Bloom The Anxiety of Influence (1973) constituyó un hito, enfocado no en el influyente autor de mayor edad e influencia, sino en el autor joven y ansioso, desgarrado entre la admiración por un clásico y el impulso hacia la originalidad.

31 Thomas Aquinas, Summa theologiae, Cologne, Hieratus, 1604, 1a, q. 75, a. $5 ; 3$ a, q. 5 .

32 Henri de Lubac, Exegèse médiévale: les quatre sens de l'écriture (4 vols.), Paris, Aubier, 1959-64, 1: p. 290-304.

33 Werner Jaeger, Early Christianity and Greek Paideia, Cambridge, Harvard University Press, 1962.

34 "Ravissant les biens d'Egypte pour en jouir": Certeau, L'Invention du quotidien, op. cit. p. 292
Otro enfoque se originó no en la literatura sino en la antropología social. En los años cincuenta, Edward Evans-Pritchard describió la tarea de interpretar una cultura desde otra como "traducción cultural". Su enfoque ha sido criticado por no considerar las relaciones de poder involucradas en los procesos de traducción, pero el mismo comentario ha sido hecho sobre las traducciones entre lenguas, por lo que la analogía todavía se sostiene..$^{35} \mathrm{El}$ concepto de Evans-Pritchard se extendió gradualmente, desde considerar a la antropología como un estudio de traducciones hasta a referirse a los actos de traducción que se llevan a cabo al estudiar una determinada cultura. ${ }^{36}$ Un vívido ejemplo de este último proceso proviene de la antropóloga estadounidense Laura Bohannan, quien describe una sesión de narración de cuentos en un poblado de África occidental, en el que realizaba su trabajo de campo. Cuando llegó su turno de contar una historia, decidió resumir la trama de Hamlet. Sin embargo, los ancianos de la aldea la interrumpían y le 'corregían' la historia. En el proceso ellos localizaban Hamlet, lo adaptaban a su entorno y transformaban la obra en un cuento folclórico de África occidental. ${ }^{37}$

Bohannan escribía en los años sesenta cuando la idea de traducción cultural estaba más o menos confinada a la antropología. Por supuesto desde entonces su uso se ha difundido a otras disciplinas. El argumento según el cual todos somos productores cuando adaptamos ideas o artefactos a nuevos propósitos se hizo cada vez más frecuente en el momento que se nombró como 'translational turn' en los años noventa. Así como la traducción es vista como un tipo de negociación, una negociación puede ser considerada como una forma de traducción. ${ }^{38}$

En los estudios sobre religión, por ejemplo, se ha vuelto casi un lugar común presentar a los misioneros como traductores entre sistemas culturales. Ellos afrontaron un dilema común al dilema de los traductores, al caminar por la cuerda floja entre la fidelidad al texto original y su inteligibilidad para una nueva audiencia. Por ejemplo, en su estudio sobre Maurice Leenhardt, un misionero protestante francés en Nueva Caledonia que más tarde se convirtió en antropólogo, James Clifford escribió sobre "la idea de un cristianismo traducible entre culturas" .39

35 Talal Asad, "The Concept of Cultural Translation", en James Clifford y George Marcus (eds.), Writing Culture, Berkeley, University of California Press, 1986, pp. 141-164.

36 Edward E. Evans-Pritchard, Social Anthropology, London, Routledge, 1951, pp. 81-82; Thomas O. Beidelman (ed.), The Translation of Cultures, London, Tavistock, 1970.

37 Laura Bohannan, "Shakespeare in the Bush" (1966), en David S. Kaston (ed.), Critical Essays on Shakespeare's Hamlet, New York, Prentice-Hall, 1995.

38 Anthony Pym, "Negotiation Theory as an Approach to Translation History: an Inductive Lesson from 15thc Castille", en Yves Gambier and Jorna Tommola (eds.), Translation and Knowledge, Turku, Grafia Oy, 1993, pp. 27-39; Umberto Eco, Mouse or Rat? Translation as Negotiation, London, Weidenfeld and Nicolson, 1993.

39 James Clifford, Person and Myth: Maurice Leenhardt in the Melanesian World, Berkeley, University of California Press, 1982, p. 79. Ver Cristina Pompa, Religião como tradução. Missionários, Tupi e Tapuia no 
Para un ejemplo vívido, o una serie de ejemplos de este proceso de caminar por la cuerda floja, uno podría tomar el caso de Matteo Ricci, el jesuita italiano que se convirtió en una figura líder de la misión a China en el siglo dieciséis. En primer lugar, Ricci tuvo que traducirse a sí mismo. Comenzó vistiendo las túnicas de un monje budista que lo hizo aceptable en su nuevo ambiente al precio de ser tratado como alguien de bajo estatus. Peor todavía para la misión, los chinos percibían a la doctrina católica "a través de los lentes del budismo" y, por ejemplo, veían las imágenes de la Virgen María como representaciones de la diosa local Guanyin. ${ }^{40}$

Al reaccionar contra lo que él percibió que había sido una mala traducción cultural, Ricci dejó sus túnicas de monje y se puso las ropas de un erudito chino. Además comenzó a presentar al cristianismo como consistente con las ideas de Confucio, en sus palabras, "acomodándolo", a la cultura local. De ahí que les describiera a los chinos al Dios cristiano como "Señor del Cielo" (Tianzhu) o "Alto Soberano" (Shangdi), ya que las referencias al Cielo y al Alto Soberano aparecían en los textos clásicos del confucionismo. Ricci también se enfrentó a la difícil decisión de tratar el culto a los antepasados o bien como una forma de religión, en cuyo caso sus conversos tendrían que abandonarlo, o bien como una costumbre social, en cuyo caso podría mantenerlo. Ricci y sus sucesores en la misión eligieron esta última opción. Así, tuvieron más conversos al precio de ser denunciados por los misioneros rivales como no cristianos. ${ }^{41}$

Otro campo en el que la idea de traducción cultural se ha vuelto prominente es el de los estudios cinematográficos. Después de todo, en el ámbito del cine, las adaptaciones de textos resultan por demás comunes, e incluso algunas adaptaciones involucran no sólo un cambio de medio, sino también una transferencia de un país o un período a otro. ${ }^{42}$ Un ejemplo espectacular de transferencia a través del espacio, el tiempo y la cultura fue Throne of Blood (1957), de Akira Kurosawa, un film que traduce el Macbeth de Shakespeare (en sí mismo una traducción cultural del siglo once escosés a la Inglaterra jacobina) al mundo feudal Japón del siglo dieciséis.

La metáfora de la traducción tiene la gran ventaja de enfatizar la acción y poner la atención en el trabajo de adaptación ejercido por los mediadores entre culturas, disciplinas académicas,

Brasil colonial, São Paulo, ANPOCS, 2003.

40 Ronnie Po-chia Hsia, A Jesuit in the Forbidden City: Matteo Ricci, 1552-1610, New York, Oxford University Press, 2010, pp. 92 y 168. Ve Qiong Zhang, "The Politics of Cultural Translation and Interpretation in the Early Jesuit Mission," en Lydia Liu (ed.), Tokens of Exchange: the Problem of Translation in Global Circulation, Durham, NC, 1999, pp. 74-106.

41 Po-chia Hsia, A Jesuit in the Forbidden City, op. cit. pp. 81, 138, 158, y pp. 293-8.

42 Brian McFarlane, Novel to Film: an Introduction to the Theory of Adaptation, Oxford, Clarendon Press, 1996, especialmente pp. 8-10. etc. También les recuerda a los estudios de recepción que deben buscar qué es lo que "se pierde en la traducción" o qué es lo distorsionado: como escribió Cervantes, leer un texto traducido es "como ver tapices flamencos desde el lado equivocado".43

\section{4}

La idea de recepción, tal como ha sido elaborada y desarrollada durante la última generación, ofrece perspectivas y oportunidades a la historia intelectual, pero también trae los problemas que vienen con ella. La teoría de la recepción todavía conlleva las marcas de los contextos en los que se originó. En el caso de Jauss e Iser, este contexto parte de los estudios literarios alemanes de finales de los años sesenta, cuando algunos teóricos que pertenecían a una nueva generación se rebelaron contra otra anterior. De nuevo, las ideas de Harold Bloom sobre la ansiedad de la influencia, presentadas como universales, fueron formuladas en relación a los poetas de los siglos xix y xx. Sin embargo, estas ideas todavía resultan útiles para los historiadores si son adaptadas adecuadamente. Un estudio sobre los poetas del Renacimiento, por ejemplo, tendría que comenzar por reconocer que las actitudes de imitación no fueron las mismas que, más adelante, tomaron los románticos tardíos y los post-románticos.

Los estudios de recepción enriquecen a la historia intelectual al alentar a los académicos del campo a no limitarse a la reconstrucción de las intenciones de los principales pensadores, sino a formular una gama mucho más amplia de interrogantes sobre recontextualizaciones, respuestas, usos y otras preguntas en este sentido. Un enfoque comparativo resulta particularmente esclarecedor, por ejemplo, al centrarse en las cálidas o frías recepciones de un mismo texto o autor en diferentes países o en la de diferentes autores en un mismo país. Sin embargo, incluso en el caso de un texto en un mismo país puede ser prudente hablar de 'recepciones' en plural: como por ejemplo, las respuestas británicas divididas a los Satanic Verses de Salman Rushdie cuando aparecieron por primera vez en 1988.

Como de costumbre, nuevas oportunidades vienen acompañadas de nuevos problemas, dos en particular. En primer lugar, ¿qué define a la recepción? ¿En qué medida depende de afinidades, resonancias o de la fusión de horizontes de expectativas? Las aparentes afinidades, o el reconocimiento de sí mismo en el otro, han jugado un papel importante en las relaciones culturales entre Japón y el occidente. Por ejemplo, el interés por el arte japonés en Francia hacia finales del siglo XIX estuvo relacionado con el auge del impresionismo. Camile Pissarro, un entusiasta de la obra de Utagawa Hiroshige, lo describió como "un maravillosos

43 Miguel de Cervantes, Don Quixote (ed. Francisco Rico), Barcelona, Instituto Cervantes, 1999, parte 2, cap. 62. 
impresionista". A la inversa, el historiador del arte de origen japonés Yukio Yashiro publicó un libro sobre Botticelli en 1925 en el que comparaba su trabajo con el de artistas japoneses como Kitagawa Utamaro.

En la medida en que la recepción puede adoptar diferentes formas, el bibliógrafo Don McKenzie ha señalado la importancia de la disposición física de los libros (el tamaño de las páginas, la fuente del tipo, etc.) como parte del mensaje del texto. Como él dijo, "las formas generan significados". En un sentido similar, como hemos visto, el crítico francés Gérard Genette enfatizó el papel de los 'paratextos' —dedicatorias, epígrafes, introducciones, prefacios, ilustraciones, notas, índices, etc.- como otros tantos medios, a disposición del autor, los redactores, los editores y las editoriales, capaces de generar distintas respuestas en los lectores. También las reseñas de libros han colaborado a conformar las expectativas de otros lectores (y de algunos no lectores), desde la invención del género en las revistas científicas de finales del siglo XVII hasta la actualidad.4

Cuando se traduce un texto, el traductor tiene el poder de dar forma al proceso de recepción, especialmente cuando se adopta un modo de domesticar la traducción. Esto fue en general el caso de los primeros traductores modernos, que se permitían a sí mismos omitir y añadir pasajes e incluso mover partes del texto de un lugar a otro. ${ }^{45}$ Cuando el Arte de la guerra de Maquiavelo fue traducido al español, el diálogo se desplazó de Italia a España mientras que los oradores, originalmente el romano Fabrizio Colonna y el fiorentino Cosimo Rucellai, se convirtieron en dos españoles, el Gran Capitán Gonzalo Fernández de Córdoba y el Duque de Najara. ¿Por qué? Presumiblemente porque los lectores españoles en la era de los famosos tercios no hubieran esperado aprender nada de los italianos sobre la guerra. Tal idea estaba más allá de su horizonte, y entonces semejante traducción cultural resultaba necesaria.

El proceso de recepción de Utopía de Tomás Moro conforma un caso particularmente interesante porque podía ser descrito, al igual que Cortegiano de Castiglione, como texto fundante de un género al cual no pertenecía. Utopía no fue escrito como un anteproyecto de una sociedad ideal como tampoco el Cortegiano fue escrito como una guía de buenos modales. Estos fueron transformados en este tipo de anteproyectos o guías por sus impresores, editores, traductores e imitadores. Un reciente estudio colectivo sobre Utopía de Moro en la temprana modernidad europea muestra muy claramente como las ediciones y las traducciones pueden ser analizadas para revelar la historia de su recepción, basándose en un cuidadoso análisis conjunto de las traducciones

44 Donald F. McKenzie, Bibliography and the Sociology of Texts, London, British Library, 1986; Gérard Genette, Seuils, Paris, Seuil, 1987.

45 Peter Burke, "Cultures of Translation in Early Modern Europe", en Peter Burke y R. Po-chia Hsia (eds.), Cultural Translation in Early Modern Europe, Cambridge, Cambridge University Press, 2007, pp. 7-38. alemanas, italianas, francesas, holandesas, francesas, inglesas y españolas, con sus paratextos que aparecen, desaparecen y en ocasiones reaparecen a través de las distintas ediciones. ${ }^{46}$ Sería bueno observar los principales trabajos de Bodin tratados de esta misma manera.

Otro esclarecedor estudio de caso concierne a la recepción de los escritos de Adam Ferguson en la Alemania del siglo XVIII, que se concentraron en los problemas de traducción de un discurso (en el sentido de una red interconectada de conceptos) en otro. Por ejemplo, cuando el concepto clave de Ferguson sociedad civil se convirtió en bürgerliche Gesellschaft, su significado cambió porque la frase en alemán, a diferencia de la inglesa, "no contenía un espacio definido para el ciudadano". Nuevamente, el uso de términos como Seele (soul) para traducir "mind" movía el texto en dirección al pietismo alemán. ${ }^{47}$

De manera más general, puede sostenerse que la ley de las consecuencias no intencionadas en historia es tan válida en la historia de la recepción como en cualquier otro lado, y que incluso si los traductores intentan ser fieles al original, el lenguaje y de hecho la cultura a la cual se traduce funcionan como condiciones para su trabajo, con un producto híbrido como resultado.

Un producto híbrido de este tipo se considera a menudo como una mala traducción, lo que plantea un segundo problema llamado el "problema del mal o de la desviación": que refiere no sólo a una mala traducción, sino también al malentendido, la mala lectura, la mala interpretación o el mal uso —aunque además se califique también estos productos como 'creativos'. En la última generación, una serie de estudios se han dedicado a este tema. Por ejemplo, Harold Bloom dedicó un libro a lo que llamó mala lectura. El crítico brasileño Roberto Schwarz escribió el famoso ensayo "Las ideas fuera de lugar" (As ideias fora do lugar). El estudio de las respuestas alemanas a los escritos de Adam Ferguson, citado anteriormente, fue descrito por su autor como un estudio de 'mala recepción'. 48

El ensayo sobre las ideas fuera de lugar se centra en lo que el autor Llama "la disparidad entre la sociedad esclavista de Brasil y los principios del liberalismo europeo". El texto ofrece una brillante crítica de la cultura brasileña, pero falla en reconocer que el problema no es sólo de los brasileños, en un momento de

46 Terence Cave (ed.), Thomas More's Utopia in Early Modern Europe, Manchester, Manchester University Press, 2008.

47 Fania Oz-Salzberger, Translating the Enlightenment: Scottish Civic Discourse in Eighteenth-Century Germany, Oxford, Clarendon Press, 1995, pp. 142 y 144, y pp. 158-9.

48 Harold Bloom, A Map of Misreading, New York, Oxford University Press, 1975; Roberto Schwarz, "Misplaced Ideas", en John Gledson (ed.), Misplaced Ideas: Essays on Brazilian Culture (1977), London, Verso, 1992, pp. 19-32; Oz-Salzberger, Translating the Enlightenment, pp. 2-3 у pp. $77-85$. 
especial "movilidad cultural" ${ }^{49}$ Siempre que las ideas son llevadas a nuevos lugares, están necesariamente 'fuera de lugar'. Una vez más, al hablar de 'mala recepción', como hace el estudio de Ferguson, implica que una correcta recepción es posible, incluso cuando toda recepción involucra una adaptación y recontextualización.

El estudio de Ferguson también trae el viejo problema de la traducibilidad, entre otros, discutido por Iser. ${ }^{50}$ En muchos idiomas, quizás en todos, hay ciertas palabras que los nativos consideran intraducibles. Tienen un aura especial, una riqueza de asociaciones que se pierde en la traducción. Desafortunadamente para los extranjeros, estos términos usualmente juegan un rol central en determinada cultura, tanto que Rushdie puede remarcar en otras de sus novelas, Shame, que "para entender otra cultura, hay que mirar sus palabras intraducibles".

El problema resulta tan agudo que ha llevado al autor de un penetrante estudio sobre traducción a describir esa actividad como una "apuesta"; a hablar de términos equivalentes en diferentes idiomas como "construidos" en lugar de encontrados; e incluso a abandonar por completo el concepto central, sustituyendo "traducción" por práctica "translingüística", definida ésta como "el proceso por el que nuevas palabras, significados, discursos y modos de representación surgen, circulan y adquieren legitimidad dentro de la lengua anfitriona debido al contacto/colaboración de ésta con la lengua invitada o a pesar de ello". ${ }^{51}$ De manera similar, los historiadores y filósofos de la ciencia, en particular Thomas Kuhn, han argumentado que las teorías o paradigmas sucesivos resultaban inconmensurables, e involucraban cambios en la visión del mundo que hacían imposible la comparación racional. 52

Sin embargo, de nuevo, una oposición binaria, en este caso entre lo traducible y lo intraducible, lo conmensurable e inconmensurable, que simplifica excesivamente una situación compleja. Nos sirve más pensar en términos de un mejor o peor ajuste, o de una mayor o menor traducibilidad o proporcionalidad, entre palabras, idiomas y culturas. Esta conclusión se ha visto refrendada por un estudio ejemplar sobre el Japón del siglo XIX inspirado en parte en la obra de Koselleck. ${ }^{53}$

49 Elias J. Palti, "The Problem of 'Misplaced Ideas' Revisited", en Journal of the History of Ideas n 67, 2006, pp. 149-79; Stephen Greenblatt et al., Cultural Mobility: a Manifesto, Cambridge, Cambridge University Press, 2010.

50 Budick and Iser, Translatability, op. cit..

51 Lydia H. Liu, Translingual Practice, Stanford, Stanford University Press, 1995, p. 26; Lydia H. Liu (ed.), Tokens of Exchange, Durham, NC, Duke University Press, 1999, p. 5 y 34.

52 Thomas S. Kuhn, The Structure of Scientific Revolutions, Chicago, University of Chicago Press, 1962, pp. 110-11.

53 Douglas Howland, "Translating Liberty in Nineteenth-Century Japan," en Journal of the History of Ideas, $n^{\circ} 62$, 2001, pp. 161-81. Ver Howland Translating the West: Language and Political Reason in Nineteenth-Century Japan, Honolulu, University of Hawaii Press, 2002. Ver Jörn
Después de 1868, los nuevos gobernantes de Japón se dispusieron a modernizar el país siguiendo modelos occidentales, en parte para protegerse así mismos de Occidente. Los intelectuales prestaron una mano en el proceso, traduciendo determinados libros del inglés al japonés, entre otros, los trabajos de Charles Darwin, T. H. Huxley, Herbert Spencer y John Stuart Mill, de quien el ensayo On Liberty fue traducido en 1871. En el caso de Mill, el problema central fue la traducción de la misma palabra "liberty". El traductor buscó un término equivalente en la tradición japonesa y eligió jiyu, una palabra que ya había sido usada en los tempranos tiempos modernos para traducir el vocablo latino libertas y el holandés vrijheid. Sin embargo, jiyu traía una fuerte connotación negativa, asociada al egoísmo y la astucia en una cultura que, a diferencia de la occidental, favorece lo colectivo sobre lo individual. Este ejemplo revela con una claridad ejemplar que ciertas distinciones culturales conforman obstáculos lingüísticos para una traducción exitosa.

Es probable que los primeros lectores japoneses no hayan comprendido la alabanza de la libertad de Mill. Por otro lado, sus sucesores probablemente lo entendieron mejor una vez que el nuevo significado de jiyu se hubiera establecido. Generalizando, tal vez precipitadamente, a partir de este ejemplo, se podría sugerir que cuando las palabras extranjeras ingresan en un campo semántico dado es probable que se domestiquen, transformadas por la fuerza de los otros términos en el campo. ${ }^{54}$ Sin embargo, en el transcurso del tiempo, el campo en sí puede ser transformado por estas recién llegadas.

Al hacer esta sugerencia, yo mismo estoy reempleando las ideas de un distinguido antropólogo, Marshall Sahlins, cuyas reflexiones sobre el impacto del Capitán Cook, y más generalmente de la cultura inglesa, en Hawai desde las últimas décadas del siglo XVIII en adelante distinguió dos fases. En la primera fase, los eventos que tomaron lugar en Hawai fueron "ordenados por la cultura". Los hawaianos vieron la visita de Cook en los términos de sus propias tradiciones culturales, "reproduciendo" así en el contacto con otra cultura la imagen que ellos tenían de la suya propia (o, de acuerdo al uso del lenguaje en este capítulo, creando una traducción cultural). Sin embargo, en el curso de este reordenamiento o reproducción, la cultura hawaiana fue transformada o reordenada. ${ }^{55}$ Lo que parece haber sucedido en el caso japonés era que en una primera fase la idea de libertad fue absorbida en un campo lingüístico tradicional. Pero, en cierto momento, una vez que se superó un umbral crítico, el campo mismo se reordenó.

Leonhard, "Language, Experience and Translation: towards a comparative dimension", en Javier Fernández Sebastián (ed.), Political Concepts and Time, Santander, Cantabria University Press, 2011, pp. 245-72.

54 Howland, Translating the West, op. cit., pp. 28-9.

55 Marshall Sahlins, Islands of History, Chicago, University of Chicago Press, 1985, pp. 136-56. 
Como sugieren estos ejemplos, la traducción, ya sea entre lenguas o entre culturas, no es un tema marginal para los historiadores, aunque sólo recientemente haya entrado en su agenda. Si "el pasado es un país extranjero", los historiadores son todos traductores del lenguaje del pasado al del presente. Incluso, uno podría decir que ésta es su raison d'être. Como otros traductores, ellos enfrentan un dilema central, entre la fidelidad al lenguaje que están traduciendo y la inteligibilidad del público para el cual están traduciendo. En otras palabras, el dilema no queda confinado a la historia de la recepción: constituye a la empresa de la historia en sí misma.

\section{Referencias Bibliográficas}

Aquinas, Thomas, Summa theologiae, Cologne, Hieratus, 1604, 1a, q. 75 , a. 5; 3a, q. 5 .

Bailey, Cyril (ed.), The Legacy of Rome, Oxford, Clarendon Press, 1923.

Bataillon, Marcel, Erasme en Espagne (1937), Geneva, Droz, 1991, p. 846

Baxandall, Michael, Patterns of Intention, New Haven, Yale University Press, 1985, pp. 58-9.

Beidelman, Thomas O. (ed.), The Translation of Cultures, London, Tavistock, 1970.

Bloom, Harold, A Map of Misreading, New York, Oxford University Press, 1975

Bohannan, Laura, "Shakespeare in the Bush" (1966), en David S. Kaston (ed.), Critical Essays on Shakespeare's Hamlet, New York, Prentice-Hall, 1995.

Boutcher, Warren, "Marginal Commentaries: the Cultural Transmission of Montaigne's Essais in Shakespeare's England", en Pierre Kapitaniak y Jean-Marie Maguin (eds.), Shakespeare et Montaigne, Paris, Société Française Shakespeare, 2003, pp. 13-27.

Brady, Thomas A., The Reception of Egyptian Cults by the Greeks, Columbia, University of Missouri, 1935.

Brunner, Otto, Conze, Werner y Koselleck, Reinhart, (eds.), Geschichtliche Grundbegriffe (8 vols.), Stuttgart, Klett, 1972, p. 97.

Burke, Peter, "Cultures of Translation in Early Modern Europe", en Peter Burke y R. Po-chia Hsia (eds.), Cultural Translation in Early Modern Europe, Cambridge, Cambridge University Press,
2007, pp. 7-38.

Burke, Peter, The Fortunes of the Courtier, Cambridge, Polity Press, 1995.

Cavallo, Guglielmo y Chartier, Roger (eds.), A History of Reading in the West (trad. Lydia G. Cochrane), Amherst, University of Massachusetts Press, 1999.

Cave, Terence (ed.), Thomas More's Utopia in Early Modern Europe, Manchester, Manchester University Press, 2008.

Centre Interuniversitaire de recherche sur la Renaissance italienne, Réécritures: commentaires, parodies, variations dans la literature italienne de la Renaissance (3 vols.), Paris, Université de la Sorbonne Nouvelle, 1983, p. 7.

Cervantes, Miguel, Don Quixote (ed. Francisco Rico), Barcelona, Instituto Cervantes, 1999, parte 2, cap. 62.

Chartier, Roger, Cultural History between Practices and Representations (trad. Lydia G. Cochrane), Cambridge, Polity Press, 1988, pp. 40-1.

Clifford, James y Marcus, George (eds.), Writing Culture, Berkeley, University of California Press, 1986, pp. 141-164.

Clifford, James, Person and Myth: Maurice Leenhardt in the Melanesian World, Berkeley, University of California Press, 1982, p. 79.

Collingwood, Robin G., The Idea of Nature, Oxford, Oxford University Press, 1945, p. 128.

Cremeans, Charles D., The Reception of Calvinistic Thought in England, Urbana, University of Illinois Press, 1949.

Dale B. J. Randall y Jackson C. Boswell, Cervantes in Seventeenth-Century England: the Tapestry Turned, Oxford, Oxford University Press, 2009, pp. xv, xxxvii.

Darnton, Robert "History of Reading," en Peter Burke (ed.), New Perspectives on Historical Writing, Cambridge, Polity Press, 2001, pp. $157-86$

De Certeau, Michel, L'invention du quotidien, Paris, Union Générale d'Éditions, 1980.

De Lachenal, Lucilla, Spolia: uso e reimpiego dell'antico dal III al XIV secolo, Milan, Longanesi, 1995.

De Lubac, Henri, Exegèse médiévale: les quatre sens de l'écritu- 
re (4 vols.), Paris, Aubier, 1959-64, 1: p. 290-304.

Eco, Umberto, Mouse or Rat? Translation as Negotiation, London, Weidenfeld and Nicolson, 1993.

Espagne, Michel, Les Transferts culturels franco-allemands, $\mathrm{Pa}$ ris, Presses Universitaires de France, 1999.

Evans-Pritchard, Edward E., Social Anthropology, London, Routledge, 1951, pp. 81-82.

Evett, Elisa, (ed.), The Critical Reception of Japanese Art in Late Nineteenth-Century Europe, Epping, Bowker, 1982.

F. Glick, Thomas (ed.), The Comparative Reception of Darwinism, Austin, University of Texas Press, 1974.

Farago, Claire (ed.), Reframing the Renaissance, New Haven, Yale University Press, 1995.

Febvre, Lucien, "La Première Renaissance française," en Pour une histoire à part entière, Paris, Sevpen, 1962, pp. 529-603.

Feingold, Mordechai, Freeman, Joseph S. y Rother, Wolfgang (eds.), The Influence of Petrus Ramus, Basel, Schwabe, 2001.

Femia, Joseph V. (ed.), The Machiavellian Legacy, Basingstoke, Macmillan, 1998.

Ford, Philip, De Troie à Ithaque; réception des épopées homériques à la Renaissance, Geneva, Droz, 2007.

Gingerich, Owen, The Book Nobody Read: Chasing the Revolutions of Nicolaus Copernicus, New York, Walker, 2004.

Goldie, Mark (ed.), The Reception of Locke's Politics, London, Pickering and Chatto, 1999.

Grafton, Anthony, Most, Glenn W. y Settis, Salvatore (eds.), The Classical Tradition, Cambridge, Harvard University Press, 2010.

Graham, Allen, Intertextuality, London, Routledge, 2000, especialmente: pp. 30-60 y pp. 97-115.

Greenblatt, Stephen, Cultural Mobility: a Manifesto, Cambridge, Cambridge University Press, 2010.

H. Liu, Lydia (ed.), Tokens of Exchange, Durham, NC, Duke University Press, 1999, p. 5 y 34.

H. Liu, Lydia, Translingual Practice, Stanford, Stanford University Press, 1995, p. 26.
Hauhart, William Frederic, The Reception of Goethe's Faust in England in the First Half of the 19th Century, New York, Columbia University Press, 1909.

Herrmann, Max, Die Reception des Humanismus in Nürnberg, Berlin, Wiedmann, 1898

Holub, Robert C., Reception Theory: a Critical Introduction, London, Methuen, 1984.

Horowitz, Maryanne C., Cruz, Anne J., y Furman, Wendy A. (eds.), Renaissance Rereadings, Urbana, University of Illinois Press, 1988.

Howland, Douglas, "Translating Liberty in Nineteenth-Century Japan," en Journal of the History of Ideas, n 62, 2001, pp. 161-81.

Howland, Douglas, Translating the West: Language and Political Reason in Nineteenth-Century Japan, Honolulu, University of Hawaii Press, 2002

Immisch, Otto, Das Nachleben der Antike, Leipzig, Dieterich, 1919.

Iser, Wolfgang, Akt des Lesens; Susan Suleiman e Inge Crosman (eds.), The Reader in the Text, Princeton, Princeton University Press, 1980.

Iser, Wolfgang, Der Akt des Lesens: Theorie ästhetischer Wirkung, Munich, Fink, 1976.

Jaeger, Werner, Early Christianity and Greek Paideia, Cambridge, Harvard University Press, 1962.

Jauregui, Carlos A., Canibalia. Canibalismo, calibanismo, antropofagia cultural y consumo en América Latina, Madrid, Iberoamericana, 2008.

Jauss, Hans-Robert, Literaturgeschichte als Provokation, Frankfurt, Suhrkampf, 1970.

Johnson, James $\mathrm{H}$., Listening in Paris: a cultural history, Berkeley, University of California Press, 1995.

Jörn, Leonhard, "Language, Experience and Translation: towards a comparative dimension", en Javier Fernández Sebastián (ed.), Political Concepts and Time, Santander, Cantabria University Press, 2011, pp. 245-72.

Kemp, Wolfgang (ed.), Der Betrachter ist im Bild, Cologne, DuMont, 1985; David Freedberg, The Power of Images, Chicago, University of Chicago Press, 1989. 
Koselleck, Reinhart, Futures Past: on the Semantics of Historical Time (trad. Keith Tribe), Cambridge, MIT Press, 1985, pp. 7, $46,56,64,106,186,196-7,200,267-88$.

Kristeva, Julia, "Bakhtine, le mot, le dialogue et le roman", en Critique, $n^{\circ} 239,1967$, pp. 438-65.

Kuhn, Thomas S., The Structure of Scientific Revolutions, Chicago, University of Chicago Press, 1962, pp. 110-11.

Laband, Paul, Rede über die Bedeutung der Rezeption des römischen Rechts für das deutsche Staatsrecht, Strasbourg, University of Strasbourg, 1880.

Liebes, Tamar y Katz, Elihu, The Export of Meaning: Cross-Cultural Readings of Dallas, New York, Oxford University Press, 1990.

Livingstone, Richard W. (ed.), The Legacy of Greece, Oxford, Clarendon Press, 1921.

Lloyd, Howell A. (ed.), The Reception of Bodin, Leiden, Brill, 2013, pp. 21-37.

Luciani, Vincenzo, Francesco Guicciardini e la fortuna dell'opera sua, Florence, Olschki, 1949.

Martindale, Charles, Redeeming the Text: Latin poetry and the Hermeneutics of Reception, Cambridge, Cambridge University Press, 2003.

Martindale, Charles, y Richard F. Thomas (eds.), Classics and the Uses of Reception, Oxford, Blackwell, 2006.

Massaut, Jean-Pierre, Critique et tradition à la veille de la Réforme en France, Paris, Vrin, 1974.

McFarlane, Brian, Novel to Film: an Introduction to the Theory of Adaptation, Oxford, Clarendon Press, 1996, especialmente pp. 8-10.

McKenzie, Donald F., Bibliography and the Sociology of Texts, London, British Library, 1986; Gérard Genette, Seuils, Paris, Seuil, 1987

Oz-Salzberger, Fania, Translating the Enlightenment, pp. 2-3 y pp. $77-85$.

Oz-Salzberger, Fania, Translating the Enlightenment: Scottish Civic Discourse in Eighteenth-Century Germany, Oxford, Clarendon Press, 1995, pp. 142 y 144, y pp. 158-9.
Palti, Elias J., "The Problem of 'Misplaced Ideas' Revisited", en Journal of the History of Ideas $n^{\circ} 67,2006, p p .149-79$.

Po-chia Hsia, Ronnie, A Jesuit in the Forbidden City: Matteo Ricci, 1552-1610, New York, Oxford University Press, 2010, pp. 92 y 168.

Pompa, Cristina, Religião como tradução. Missionários, Tupi e

Tapuia no Brasil colonial, São Paulo, ANPOCS, 2003.

Price, Lawrence M., The Reception of English Literature in Germany, Berkeley, University of California Press, 1932.

Procacci, Giuliano, Studi sulla fortuna del Machiavelli, Rome, Istituto Storico Italiano per l'età moderna e contemporanea, 1965.

Pym, Anthony, "Negotiation Theory as an Approach to Translation History: an Inductive Lesson from 15thc Castille", en Yves Gambier and Jorna Tommola (eds.), Translation and Knowledge, Turku, Grafia Oy, 1993, pp. 27-39.

Reed, Henry H., Palladio's Architecture and its Influence, New York, Dover, 1980.

Ricoeur, Paul, "Appropriation", en Ricoeur, Hermeneutics and the Human Sciences: Essays on Language, Action and Interpretation, Cambridge, Cambridge University Press, 1981, pp. 182-93.

Sahlins, Marshall Islands of History, Chicago, University of Chicago Press, 1985, pp. 136-56.

Scanlon, Thomas F., The Influence of Thucydides on Sallust, Heidelberg, Winter, 1980.

Schmidt, Carl Adolf, Die Reception des Römischen Rechts in Deutschland (1868), Leipzig, Zentralantiquariat der DDR, 1969.

Schuler, Stefan, Vitruv im Mittelalter: Die Rezeption von "De Architectura" von der Antike bis in die frühe Neuzeit, Cologne, Böhlau, 1999.

Schwartz, Benjamin, "Some Polarities in Confucian Thought," en David Nivison and Arthur Wright (eds.), Confucianism in Action, Chicago, University of Chicago Press, 1959.

Schwarz, Roberto, "Misplaced Ideas", en John Gledson (ed.), Misplaced Ideas: Essays on Brazilian Culture (1977), London, Verso, 1992, pp. 19-32.

Seidel Menchi, Silvana, Erasmo in Italia: 1520-1580, Turin, BoUlati Boringhieri, 1987.

Skinner, Quentin, "Meaning and Understanding in the History of 
Ideas", en History and Theory, n 8, 1969, pp. 3-53.

Taylor, Alfred E., Platonism and its Influence, London, Harrap, 1925.

Tribe, Keith, Governing Economy: the Reformation of German Economic Discourse, 1750-1840, Cambridge, Cambridge University Press, 1988, p. 140.

Vermij, Rienk, The Calvinist Copernicans: the Reception of the New Astronomy in the Dutch Republic, 1575-1750, Amsterdam, Koninklijke Nederlandse Akademie van Wetenschappen, 2002.

Walter, Jacob, Wiliam Blakes Nachleben in der englischen Literatur des neunzehnten und zwanzigsten Jahrhunderts, Schaffhausen, Bachmann, 1927.

Worton, Michael y Still, Judith (eds.), Intertextuality: theories and practices, Manchester, Manchester University Press, 1990.

Zhang, Qiong, "The Politics of Cultural Translation and Interpretation in the Early Jesuit Mission," en Lydia Liu (ed.), Tokens of Exchange: the Problem of Translation in Global Circulation, Durham, NC, 1999, pp. 74-106.

Zuber, Roger, Les "belles infidèles" et la formation du goût classique, Paris, Colin, 1968.

\section{Resumen}

El presente artículo argumenta que el concepto de recepción (en alemán Rezeption, en francés réception, en italiano recezione, etc.) es más antiguo de lo que generalmente se piensa; que, aún así, el reciente 'giro' en esta dirección resulta muy significativo; que el concepto de 'traducción cultural' resulta útil para los estudios de recepción; que una serie de problemas asedian a los académicos que intentan rastrear la historia de la recepción de textos u otros artefactos culturales; y que, a pesar de estos problemas, este enfoque sigue siendo esclarecedor para la historia intelectual. Sobre la base de que cuanto más distantes están las dos culturas más visible se vuelve el proceso de recepción, el presente artículo privilegia ejemplos provenientes de las relaciones culturales entre Europa y el este asiático.

Palabras clave: Teoría de la recepción, Historia intelectual, Traducción cultural.

\section{Abstract: \\ History and Theory of Reception}

This article argues that the concept of reception is older than is generally thought; that, even so, the recent 'turn' in this direction is a significant one; that the concept of 'cultural translation' is useful in reception studies; that a number of problems plague scholars who try to trace the history of the reception of particular texts or other cultural artefacts; and that despite the problems, this approach continues to illuminate intellectual history. Examples from the cultural relations between Europe and East Asia will be privileged, on the grounds that the more distant two cultures are from each other, the more visible the reception process becomes.

Keywords: Reception theory, Intellectual history, Cultural translation. 